\title{
Study of Method of Denoising for Triaxial Flight Simulation Turntable Based on Generalized Minimum Variance
}

\author{
Yunlin Luo ${ }^{1, a}$, Hongqiang Sun ${ }^{2, b}$ \\ ${ }^{1}$ Aeronautical Automation college. Civil Aviation University of China, China \\ ${ }^{2}$ Aeronautical Automation college. Civil Aviation University of China, China \\ aylluo@cauc.edu.cn, bsunhongqiang_33@163.com
}

Keywords: triaxial flight simulation turntable, low-pass filter, PID, generalized minimum variance

\begin{abstract}
Aiming at dealing with the existing noise of triaxial flight simulation turntable, the paper design digital filter low-pass transmitters and generalized minimum variance control filter to process signal for random disturbance, and have test study based on outside box control system of triaxial flight simulation turntable. The test results show that we can get better control effect by adopting generalized minimum variance control rates to denoise for measured signal on triaxial flight simulation turntable, and that is much helpful to improve the noise control accuracy.
\end{abstract}

\section{Introduction}

Flight simulation turntable is one of important equipments of ground semi-entity simulation experiment on aircraft flight attitude, which has inner framework, middle framework and outer framework and three degrees of freedom, and is used to simulate the change of space attitude angle and angular of aircraft. Its main function is:

(1) To simulate aircraft's angular motion of three degrees of freedom;

(2) To receive and follow the command signal of three frame position from main simulator in simulation loop, and translate the signal into physical exercise that can be tested by sensor to provide conditions for the specimen.

High precision is one of the key performance indexes of flight turntable, and modern flight simulation turntable control system adopts computer control system generally. But test results always are affected by various interference factors and we gain not good control precision in the operation process of actual computer control system. As triaxial flight simulation turntable needs high control accuracy, which is particularly important to eliminate adverse effect caused by interference.

Any real existing dynamic system usually has different degree of uncertainty, and the uncertainty can be regarded as disturbance of a deterministic system. Basically have the following categories:

(1)The measurement of sensors of system have measurement noise;

(2)The system input contains random disturbance;

(3)The system model parameters and structure have uncertainty.

The top two classes is uncertain environmental factors or external disturbance, the third belongs to uncertain model factors or internal disturbances. Generally speaking, the problems of certain system model with only external disturbances can be fixed by random control; and as to the system model changing by time with unknown parameters, we need to estimate the model parameters first, and work out the optimal control in accordance with optimization performance index and conduct adaptive control. This article mainly discusses the interferences of top two classes, and we adopt digital low-pass filter and generalized minimum variance control to adjust and control signals. Also we verify and have compared test about denoising effect by the frame control system of triaxial flight simulation turntable. 


\section{Modeling of Control System of Triaxial Flight Simulation Turntable}

According to the parameters selected, we adopt brushless de motor with load model[3], and its transfer function is as follows:

$$
\frac{W(s)}{U(s)}=\frac{\frac{1}{\mathrm{Ke}}}{\left(\tau_{\mathrm{m}} \mathrm{s}+1\right) \cdot\left(\tau_{\mathrm{e}} \mathrm{s}+1\right)}
$$
constant.

Including $\tau_{\mathrm{m}}=(\mathrm{r} \cdot \mathrm{J}) /\left(\mathrm{K}_{\mathrm{e}} \cdot \mathrm{K}_{\mathrm{m}}\right)$ for electromechanical time constant, $\tau_{\mathrm{e}}=\mathrm{L} / \mathrm{r}$ for electrical time

The controlled object for turntable servo system is very complex, if we solve the problems that are adverse to control such as friction and torque disturbance focusing on position loop, it will be difficult to achieve the required performance. Therefore, we adjust the system before designing the position loop. The way to adjust is to introduce speed closed-loop into system to improve the dynamic and static characteristics by feedback channel and to increase the inner stiffness and improve the ability to suppress the interference. Also we introduce current loop and speed loop into turntable control system besides position loop. The current loop is implemented in PWM amplifiers and motor driver circuit. And speed closed-loop is implemented in analog-to-digital conversion card, in which the feed-back signal is gained from location signal processed by coding processing circuit. Turntable control system structure is shown in fig. 1 below.

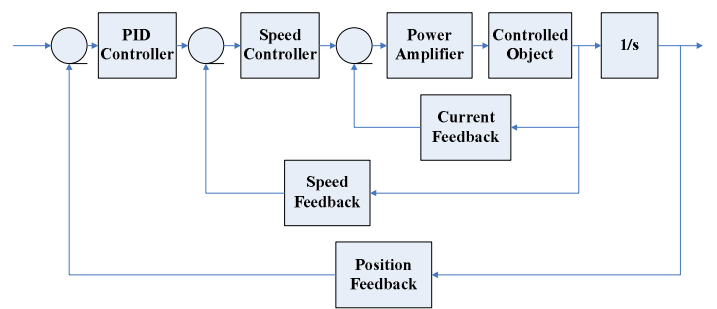

Fig. 1. Principle diagram of control system

In order to improve the stability of system and reduce the concussion, and limit the amplitude of the integral of PID control, considering the saturated error caused by the limited word length of computer and digital angle measuring device, we control the amplitude limit of error and D/A saturated output.

The model of turntable servo control system is:

$$
G(s)=\frac{1}{\mathrm{~s}} \cdot \frac{\mathrm{Kp}}{\left(\tau_{\mathrm{m}} \mathrm{s}+1\right) \cdot\left(\tau_{\mathrm{e}} \mathrm{s}+1\right)}
$$

Among them, parameter of frame motor $\tau_{\mathrm{m}}=0.5, \tau_{\mathrm{e}}=0.005$.

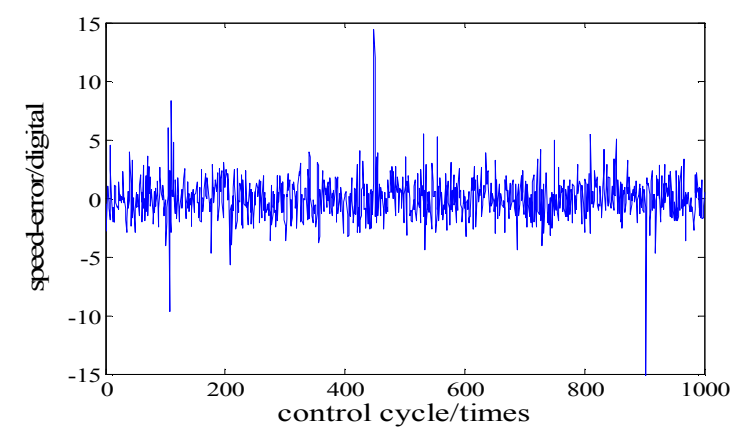

Fig. 2. Output values of system with zero input

Fig. 2. shows frame system noise measured with zero input, and the biggest random noise's fluctuation occupies \pm 15 digital quantity or so. In order to inhibit noise and interference, we add filter modules to digital feedback quantity, and realize it with computer program.

We design conventional low-pass filter and new filter based on generalized minimum variance control theory respectively to analyze disturbance signal. 


\section{Digital Low-Pass Filter}

Differential equations are used to represent the finite difference equations of ordinary hardware RC low-pass filter, so that software algorithm can be used to simulate hardware filter function. Low-pass filtering algorithm after derivation is as follows:

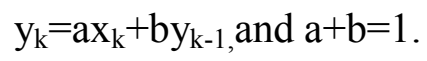

In the type, $\mathrm{x}_{\mathrm{k}}$ is the value for this collection; $\mathrm{y}_{\mathrm{k}-1}$ is the filtering output value for last time, $\mathrm{a}, \mathrm{b}$ for filter coefficients, and among them $\mathrm{a}$ is far less than $1, \mathrm{y}_{\mathrm{k}}$ for output value of this filtering. What can be seen from the type is the filter output value mainly depends on last filtering output values. This sample value is not very useful to filter output, but has somewhat fixed function. This algorithm simulates the low-pass filter function with high inertia. The cut-off frequency of filter algorithm can be calculated by next type:

$$
\mathrm{f}=\mathrm{a} / 2 \pi \mathrm{T}
$$

In the type, $a$ is a filter coefficient, $T$ is for sample interval time. Through the actual experiment of the filter effect, we select the cut-off frequency as $15 \mathrm{~Hz}$, filter order number 2 in the experiment system.

Using function yulewalk() in MATLAB Script for designing filter is as follows:

fs $=100 ; \%$ System sampling frequency $100 \mathrm{~Hz}$

filterOrder $=2$; closeFreq $=15$;

$\%$ Filter order number 2 , cut-off frequency $15 \mathrm{~Hz}$

$\mathrm{f}=[0: 0.01: 1]$;

$\mathrm{fhz}=\mathrm{f} * \mathrm{fs} / 2$;

NOne $=$ closeFreq/(fs/2)*100;

$\mathrm{H}=[$ ones $(1, \mathrm{NOne})$ zeros(1,101-NOne $)]$;

$\%$ Amplitude-frequency characteristics of ideal low-pass filter

Yulewalk method is used to construct the filters

[Bh,Ah]=yulewalk(filterOrder,f,H);

$\% \mathrm{Bh}, \mathrm{Ah}$ for filter transfer coefficient

Below are obtained amplitude-frequency characteristics curve of filter, and compare it with ideal filter.

$\mathrm{n}=256$;

$\mathrm{hh}=$ freqz $(\mathrm{Bh}, \mathrm{Ah}, \mathrm{n})$;

$\%$ Calculate the amplitude-frequency of filter.

hy=abs(hh);

plot(fhz,H);\%Amplitude-frequency characteristics of ideal filter

hold;

$\mathrm{ff}=\mathrm{fs} /(2 * \mathrm{n}) *(0: \mathrm{n}-1)$;

plot(ff,hy);\% Designed amplitude-frequency characteristics of filter

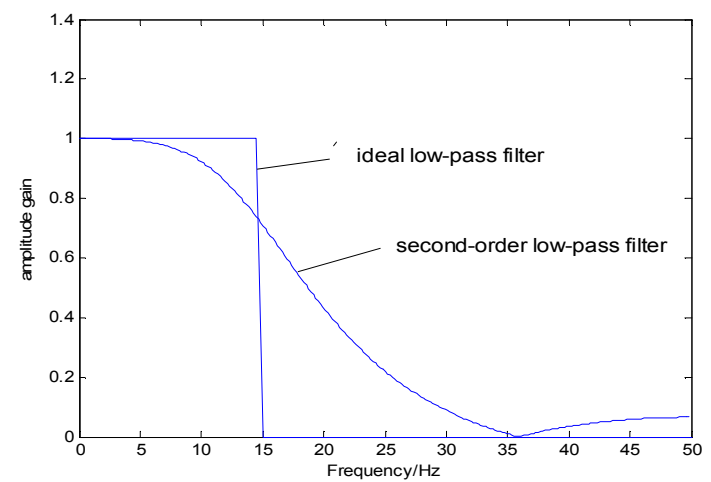

Fig. 3. Ideal low-pass filter and second-order low-pass filter 
Fig. 3 shows the comparison of designed low-pass filter and ideal low-pass filter, and the transfer function of designed second-order low-pass filter is as follows:

$$
\begin{aligned}
& \mathrm{F}\left(\mathrm{z}^{-1}\right)=\mathrm{Bh}\left(\mathrm{z}^{-1}\right) / \mathrm{Ah}\left(\mathrm{z}^{-1}\right) \\
& =\left(0.1792+0.2295 \mathrm{z}^{-1}+0.1787 \mathrm{z}^{-2}\right) /\left(1-0.6700 \mathrm{z}^{-1}+0.2572 \mathrm{z}^{-2}\right)
\end{aligned}
$$

After obtaining the molecules and common denominator coefficient of the transfer function of filter $B h=\left[b_{0}, b_{1}, \ldots, b_{n b},\right], A h=\left[a_{0}, a_{1}, \ldots, a_{n a},\right]$, we can use filter function filter () embedded in MATLAB to filter sample data, and MATLAB Script is as follows:

filterout=filter(Bh,Ah,filterin);

$\%$ filterout obtained by filtering input signal filter

Set the input for $\mathrm{x}$, output filter for $\mathrm{y}$, function filter() uses below formula to obtain output value by iterative calculation:

$$
y(n)=b_{0} x_{n}+b_{1} x_{n-1}+\ldots+b_{n b} x_{n-n b}-a_{1} y_{n-1}-a_{2} y_{n-2}-\ldots-a_{n a} y_{n-n a}
$$

Iterative calculation formula of designed low-pass filter is as follows:

$$
\mathrm{y}(\mathrm{n})=0.1792 \mathrm{x}_{\mathrm{n}}+0.2295 \mathrm{x}_{\mathrm{n}-1}+0.1787 \mathrm{x}_{\mathrm{n}-2}+0.6700 \mathrm{y}_{\mathrm{n}-1}-0.2572 \mathrm{y}_{\mathrm{n}-2}
$$

\section{Generalized Minimum Variance Control}

Fig. 3 shows control system with random disturbance, including $G\left(z^{-1}\right)$ as $Z$ domain function of controlled object, $\mathrm{v}(\mathrm{k}), \mathrm{n}(\mathrm{k})$ for random disturbance sequence, and $\mathrm{v}(\mathrm{k})$ is named process noise sequence, and $\mathrm{n}(\mathrm{k})$ measurement noise sequence. The preparation value of $\mathrm{v}(\mathrm{k})$ and $\mathrm{n}(\mathrm{k})$ in sampling time is unknown, but their statistical properties can be obtained by observing the data. Here we assume that they are all weak stationary random process, namely their statistical properties is certain. Theoretically stationary random sequence $\mathrm{v}(\mathrm{k})$ can be generated by white noise sequence $\mathrm{w}(\mathrm{k})$ through a forming filter, so that stationary random interference affecting control system can be equivalent to interference of white noise acting on system by forming filter, which brings great convenience to analyze and realize control system under random interference. According to the superposition principle of linear system, the process noise acting on the input of system and the measurement noise acting on output of system can be equivalent to the stationary random interference $\mathrm{d}(\mathrm{k})$ acting on the output of system, and the control system is as shown in fig. 4 .

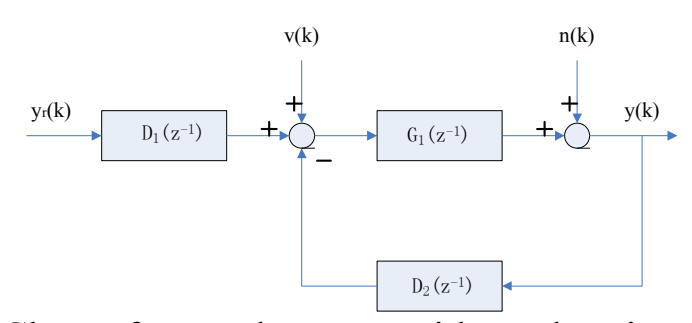

Fig. 4. Chart of control system with random interference

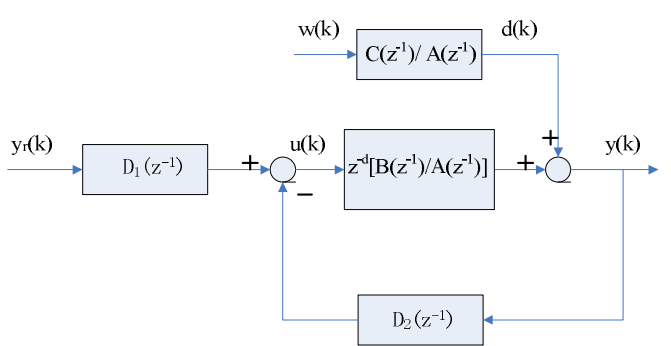

Fig. 5. Chart of standardized control system with random interference

The output of system in fig. 4. is:

$$
\mathrm{y}(\mathrm{k})=\left[\mathrm{z}^{-\mathrm{d}} \mathrm{B}\left(\mathrm{z}^{-1}\right) / \mathrm{A}\left(\mathrm{z}^{-1}\right)\right] \mathrm{u}(\mathrm{k})+\left[\mathrm{C}\left(\mathrm{z}^{-1}\right) / \mathrm{A}\left(\mathrm{z}^{-1}\right)\right] \mathrm{w}(\mathrm{k})
$$


and

$$
\begin{aligned}
& A\left(z^{-1}\right)=1+a_{1} z^{-1}+a_{2} z^{-2}+\ldots+a_{n a} z^{-n a} \\
& B\left(z^{-1}\right)=b_{0}+b_{1} z^{-1}+b_{2} z^{-2}+\ldots+b_{n b} z^{-n b} \\
& C\left(z^{-1}\right)=1+c_{1} z^{-1}+c_{2} z^{-2}+\ldots+c_{n c} z^{-n c}
\end{aligned}
$$

$\mathrm{z}^{-\mathrm{d}}$ is the delay link in system model, $\mathrm{d} \geq 1$ in computer control system. The parameters $\mathrm{A}\left(\mathrm{z}^{-1}\right), \mathrm{B}\left(\mathrm{z}^{-1}\right), \mathrm{C}\left(\mathrm{z}^{-1}\right)$ of system model can be obtained by model identification algorithm Armax. According to this algorithm, the identified model Armax of triaxial simulation turntable frame system is as follows:

$$
\begin{aligned}
& \mathrm{A}\left(\mathrm{z}^{-1}\right)=1-0.9973 \mathrm{z}^{-1} \\
& \mathrm{~B}\left(\mathrm{z}^{-1}\right)=0.0019+0.0091 \mathrm{z}^{-1} \\
& \mathrm{C}\left(\mathrm{z}^{-1}\right)=1+0.1795 \mathrm{z}^{-1} \\
& \mathrm{~d}=1
\end{aligned}
$$

Performance of characteristics of generalized minimum variance is as follows:

$$
\min J=\mathrm{E}\left[\left(\Gamma \mathrm{y}_{\mathrm{k}+\mathrm{d}}-\Psi \mathrm{r}_{\mathrm{k}}\right)^{2}+\left(\Lambda^{\prime} \mathrm{u}_{\mathrm{k}}\right)^{2}\right]
$$

and

$$
\begin{aligned}
& \Gamma=1+\gamma_{1} z^{-1}+\ldots+\gamma_{\mathrm{n} \gamma} z^{-n \gamma} \\
& \Psi=\varphi_{0}+\varphi_{1} z^{-1}+\ldots+\varphi_{n} z^{-n} \varphi \\
& \Lambda^{\prime}=\lambda_{0}+\lambda{ }_{1} z^{-1}+\ldots+\lambda{ }_{n} \lambda^{\prime} z^{-n} \lambda^{\prime}
\end{aligned}
$$

Solve the Diophantus equation:

$$
\begin{aligned}
& \Gamma \mathrm{C}=\mathrm{AF}^{\prime}+\mathrm{z}^{-\mathrm{d}} \mathrm{G} \\
& \operatorname{deg} \mathrm{F}^{\prime}=\mathrm{d}-1, \mathrm{~F}^{\prime}=1+\mathrm{f}_{1} \mathrm{z}^{-1}+\ldots+\mathrm{f}_{\mathrm{d}-1} \mathrm{q}^{-\mathrm{k}+1} \\
& \quad \mathrm{na}-1, \mathrm{na}+\mathrm{d}-1 \geq \mathrm{nc}+\mathrm{n} \gamma \\
& \operatorname{deg} \mathrm{G}=\{\mathrm{nc}+\mathrm{n} \gamma-\mathrm{d}, \mathrm{na}+\mathrm{d}-1\}<\mathrm{nc}+\mathrm{n} \gamma \\
& \text { if } \\
& \mathrm{F}_{\mathrm{K}}=\mathrm{BF}^{\prime}, \mathrm{degF}_{\mathrm{K}}=\mathrm{nb}+\mathrm{d}-1, \\
& \mathrm{H}=\Psi \mathrm{C}, \Lambda=\left(\lambda_{0} / \mathrm{b}_{0}\right) \Lambda^{\prime}, \mathrm{F}=\mathrm{F}_{\mathrm{K}}+\Lambda \mathrm{C}
\end{aligned}
$$

then obtain the control output of generalized minimum variance:

$$
\mathrm{u}_{\mathrm{k}}=(\mathrm{H} / \mathrm{F}) \mathrm{y}_{\mathrm{r}}(\mathrm{k})-(\mathrm{G} / \mathrm{F}) \mathrm{y}_{\mathrm{k}}
$$

Combined with frame control system model, a generalized minimum variance control is derived:

$$
\mathrm{u}_{\mathrm{k}}=-0.1882 \mathrm{u}(\mathrm{k}-1)+1.1773 \mathrm{y}_{\mathrm{r}}(\mathrm{k})-1.1746 \mathrm{y}(\mathrm{k})
$$

Including the $\mathrm{u}_{\mathrm{k}}$ and $\mathrm{u}_{\mathrm{k}}(\mathrm{k}-1)$ is the control quantity of first $\mathrm{k}$ and $\mathrm{k}-1$ control cycle respectively, and $\mathrm{y}(\mathrm{k})$ is the control output of first $\mathrm{k}$ control cycle, and $\mathrm{y}_{\mathrm{r}}(\mathrm{k})$ is input of system of 400 digital quantity.

In the frame control system, the speed of motor is selected as controlled variables. We adopt low-pass digital filter and generalized minimum variance control to carry out comparative experiment with PID control, and the expected input signal of system is a sine wave with cycle of $7.5 \mathrm{~s}$, amplitude digital amount of 400 . 


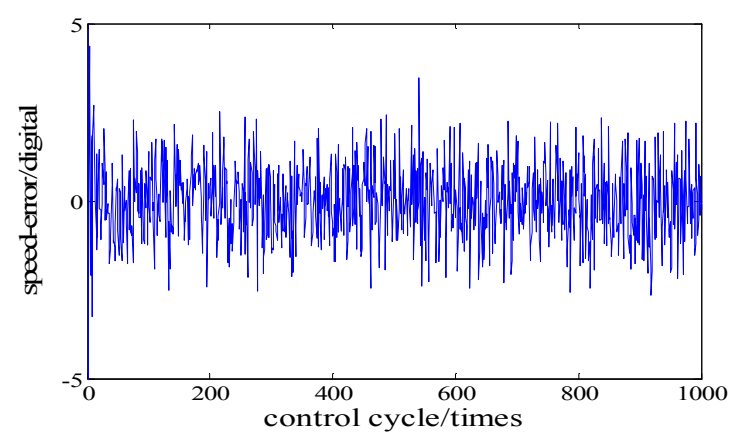

Fig. 6. System response tracking error curve using low-pass filter

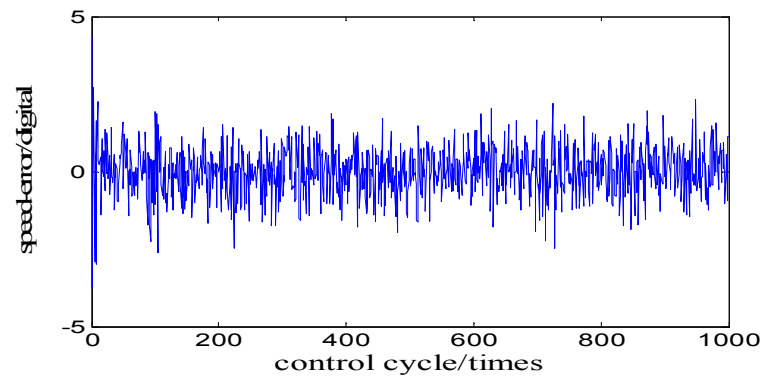

Fig. 7. System response tracking error curve using generalized minimum variance control

Fig. 6 and fig. 7 represent the tracking error curve of experimental system response. We know that fig. 1 shows the system tracking error under the action of PID controller without adopting eliminating the interference from the original system, also we can see that the maximum amplitude of system error is over \pm 15 digital quantity; fig. 6 shows the system tracking error curve by combining the signal from low-pass filter with PID controller, when the biggest error amplitude is within \pm 4 digital quantity; fig. 7 shows the system tracking error curve adopting PID control after processing with generalized minimum variance control principle, and its biggest error amplitude if plus or minus \pm 1 digital quantity. Thus, the designed second-order low-pass filter is effective to eliminate measurement noise, and improve the control precision of system to a certain extent with filter, however, using generalized minimum variance control can get the best results.

\section{Summary}

At present, the domestic triaxial flight simulation turntable always adopt the method of low-pass filter to deal with measured data, the paper studies and analyzes this method first, and then discusses how to eliminate noise and interference by bringing about generalized minimum variance control, and the results of experiment shows that the generalized minimum variance control obtains good effect in application of denoising in triaxial flight modeling turntable. Therefore, the introduction of generalized minimum variance control can not improve the ability of anti-disturbance in triaxial flight simulation turntable control system, but also has positive meaning in improving the control precision of control system.

\section{References}

[1] KROZEL J ANDRISAN I D. Intent inference and strategic path prediction[C]//AIAA Guidance, Navigation and Control Conference and Exhibit, 2005:15-18.

[2] Sciavicoo L, Siciliano B. Modeling and control of robot manipulators. London: Springer,2000,10-60

[3] Moody L. The history and future of flight control simulation. In: Proceedings of AIAA Modeling and Simulation Technologies Conference and Exhibit, Providence; AIAA, 2004. 5149-5159

[4] Martin,J.N.,Sinclair,A.J.,Cochran,J.E., "Nonlinear Tracking of Natural Mechanical Systems for HWIL Simulation,"Guidance,Navigation, and Control Conference,AIAA,Hilton Head, SC,2007.

[5] MATLAB, Software Package, Ver. 7.0,The MathWorks, Inc.,Natick, MA, 2004.

[6] Cook, M. V., Flight Dynamics Principles, Butterworth-Heinemann; 2 edition, 2007, pp. 149.M. 